Revue

d'ethnoécologie
Revue d'ethnoécologie

$14 \mid 2018$

Varia

\title{
Les termitières, un univers de chasse (nord du Cameroun)
}

Termite mounds - a hunting universe (Northern Cameroon)

\section{Christian Seignobos}

\section{(2) OpenEdition}

\section{Journals}

Édition électronique

URL : http://journals.openedition.org/ethnoecologie/3593

DOI : $10.4000 /$ ethnoecologie.3593

ISSN : 2267-2419

Éditeur

Laboratoire Eco-anthropologie et Ethnobiologie

Référence électronique

Christian Seignobos, "Les termitières, un univers de chasse (nord du Cameroun) », Revue d'ethnoécologie [En ligne], 14 | 2018, mis en ligne le 31 décembre 2018, consulté le 01 mai 2019. URL http://journals.openedition.org/ethnoecologie/3593; DOI : 10.4000/ethnoecologie.3593

Ce document a été généré automatiquement le 1 mai 2019.

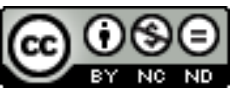

Revue d'ethnoécologie est mis à disposition selon les termes de la licence Creative Commons Attribution - Pas d'Utilisation Commerciale - Pas de Modification 4.0 International. 


\section{Les termitières, un univers de chasse (nord du Cameroun)}

Termite mounds - a hunting universe (Northern Cameroon)

\section{Christian Seignobos}

1 Le peuplement des piémonts des monts Mandara dans l'arrière-pays de Maroua relève, plus qu'ailleurs, d'imbrications de groupes, eux-mêmes très composites. Espace de repli des Giziga bwi Marva chassés par les Peuls après leur laborieuse conquête de Marva (Maroua) au début du XIX ${ }^{e}$ siècle, il s'étend depuis Dogba, au nord, jusqu'à Djébé, Kaliao, Mogoudi et la rivière Tchanaga. Selon les rapports et les cartes de l'administration coloniale la région serait un mélange mal identifié de Giziga et de Mofu. De fait, il s'agit également, dans sa partie méridionale, de communautés mowo, sans doute le fond de peuplement le plus ancien dans la région. 


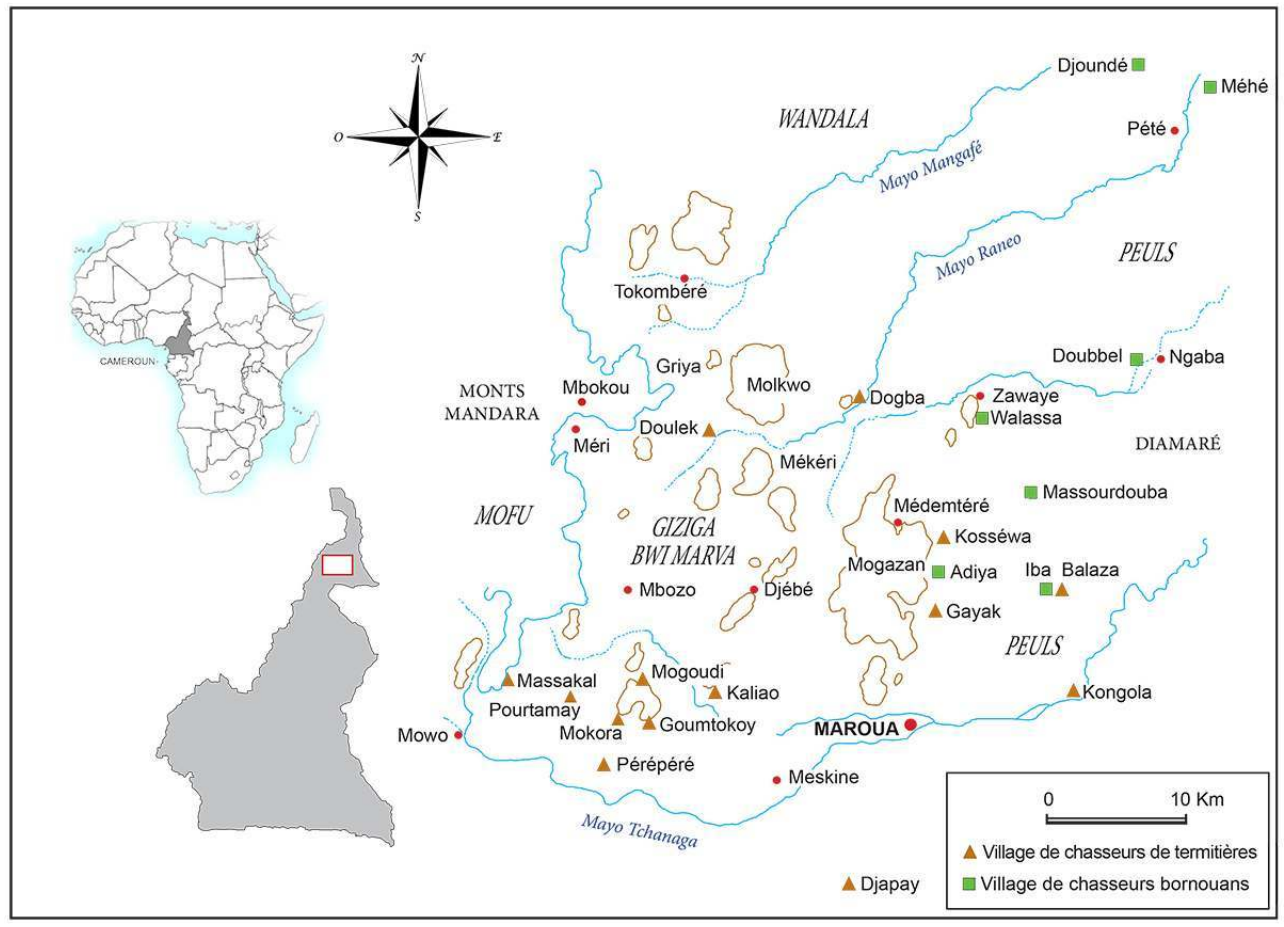

(C) L. Venot d'après C. Seignobos

2 À partir des années 1950 on enregistre sur ces piémonts une descente des montagnards mofu nord, de Mékéri, Mbokou, Molkwo... Toutefois dans ces mêmes no man's lands entre montagne et plaine, quelques colonies d'éleveurs peuls les avaient précédés de peu, comme à Mbozo. À l'est de Dogba, un autre no man's land autrement plus vaste s'étendait entre les principautés peules de Maroua et Pété et, au nord, le royaume du Wandala, avec pour frontière le mayo ${ }^{1}$ Mangafé. Longtemps très giboyeux il était anciennement parcouru par des chasseurs professionnels gamergu-kanuri venus du Bornou. Depuis les années 1950-1960 ces zones se sont peuplées de cultivateurs et la faune des grands herbivores et de leurs prédateurs n'a cessé de s'appauvrir. Elle a même disparu des piémonts où la chasse se limitait à débusquer les gibiers de terriers et plus précisément ceux qui faisaient le choix de vieilles termitières.

Nous allons d'abord présenter l'exploitation des termitières vives pour la simple et bonne raison que les récolteurs de termites représentent près de $70 \%$ de notre corpus d'une quarantaine de "chasseurs" de termitières répartis sur une douzaine de villages enquêtés ${ }^{2}$. Tous témoignent d'une connaissance cumulée sur les termitières, qu'elles soient en fonction ou dites mortes.

\section{Les termitières vives avant la désertion de leurs colonies}

Il s'agit pour l'essentiel de deux types de termitières appelées par les Giziga bwi Marva ${ }^{3}$ veye'd pour les termites rougeâtres (Odontotermes magdalena) et ilimi'd pour les gros termites noirs (Macrotermes subhyalinus). 
5 Veye'd désigne aussi leurs termitières épigées plus ou moins dressées. Manjara sont les ouvriers, bwi a manjara, les soldats qui défendent la colonie. Les départs des sexués ailés ont généralement lieu au début de la saison des pluies, au moment de la prière de l'aube ( assalaatu subaha, fflde). Cette catégorie de termites est alors dite hallal « car elle connaît la prière ", aussi les musulmans la retiennent-ils pour leur consommation.

Les Giziga bwi Marva différencient les termitières veye'd en fonction de la nature du sol : veye'd nguu wula sur les argiles à montmorillonites et veye'd nguu liigazang sur des argiles rouges caillouteuses très particulières des piémonts des collines vulcanosédimentaires du nord de Maroua.

En mai, lorsque les paysans préparent leurs champs, de nombreuses termitières sont alors signalées par une branche d'Acacia comme appropriées. Sur le piémont mofu les " propriétaires » vont habiller la superstructure de la termitière avec des litages de paille enroulés à la façon d'une toiture et qui prendront appui sur cinq ou six piquets périphériques. On peut aussi couvrir la termitière de branches maintenues entre elles par un réseau de liens d'écorce. Les arbres choisis sont ceux gardant leurs feuilles, comme Anogeissus leiocarpus et Combretum glutinosum, dont l'odeur a un effet répulsif sur les termites. Sur la base de la termitière est aménagée une rigole avec trois à quatre vasques d'argiles (mefteter) aux parois parfaitement lissées (Seignobos et al. 1996).

En juin, lors des premières pluies, les termitières sont auscultées, lorsque se prépare l'essaimage qui se réalise en une seule fois dans l'année, dans une forme d'instantanéité. Le « propriétaire » surveille la surface, l'apparition de soldats sur les bouches d'envol. On cherche à capter le bruit des ailés dans les conduits, les plus couramment répertoriés seraient d'abord "sle sle sle », puis lorsque l'essaimage approche il devient «fu fu fu » rendant ainsi compte de leur concentration. Dès lors il convient pour l'homme de se faire discret autour de l'édifice appareillé, plus un mot, on échange par sifflements. Lorsque les premiers ailés sortent, le bas des pailles est prestement dégagé. De l'eau est versée dans la rigole circulaire et des brandons, des lampes tempête, des lampes de poche et maintenant des ampoules à diodes électroluminescentes sont disposées devant chaque mefteter. Attirés par les lumières les ailés se précipitent pour amorcer de là leur envol. Adultes et enfants les balaient alors frénétiquement pour en remplir des cuvettes contenant un fond d'eau. 
Figure 2 : Termitière habillée de chaume à Massakal

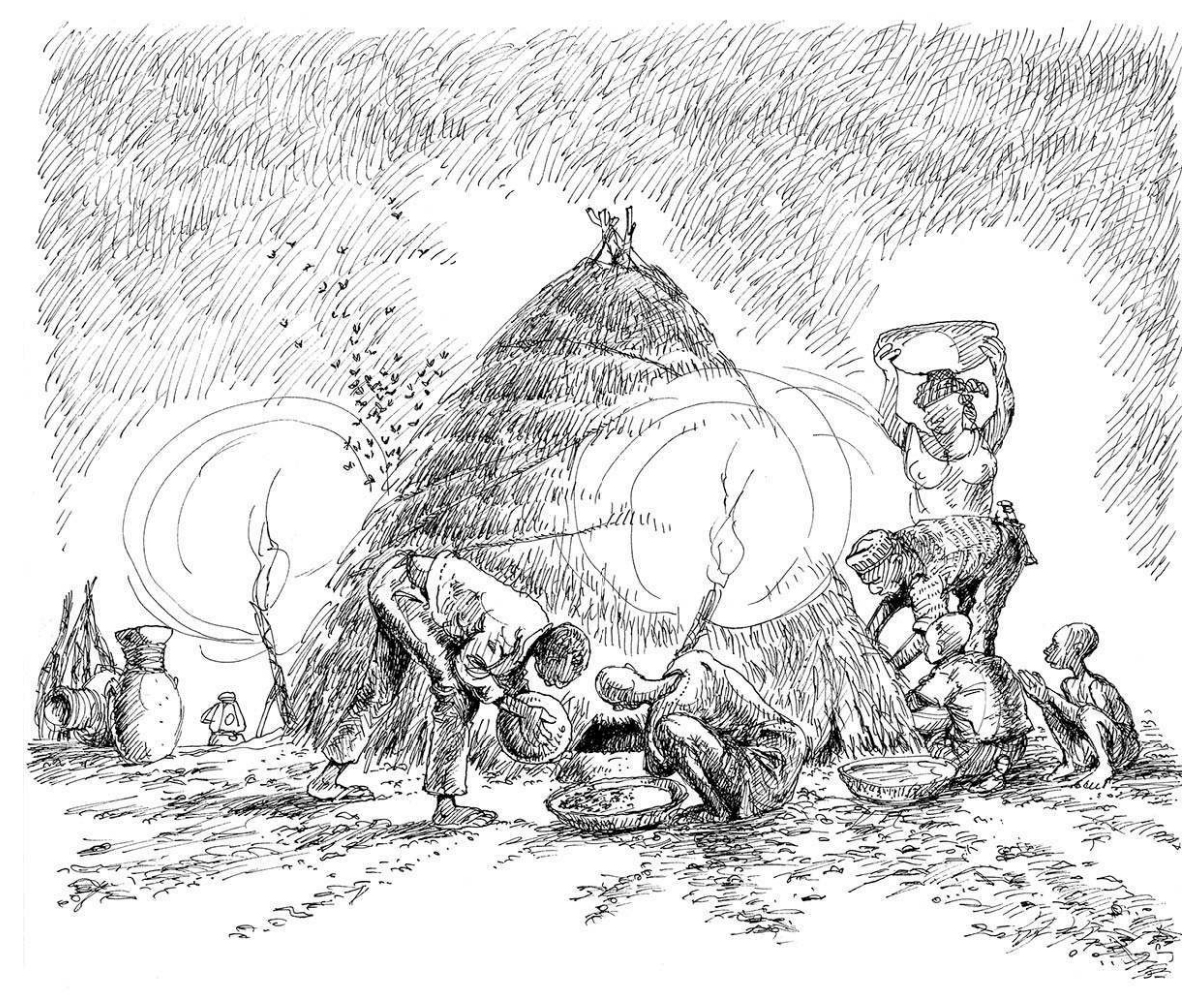

Dessin C. Seignobos

On peut aussi appareiller les termitières ilimi'd, toutefois les vols des ilimi'd seraient différents de ceux des veye'd. Leurs ailés se répandent autour de la termitière avant de s'envoler, provoquant un bruit semblable au sifflement du cobra et que l'on peut identifier à quelque distance. Les imagos de termitières voisines partiraient en même temps, multipliant ainsi leurs chances d'échapper à leurs innombrables prédateurs.

On appareille plus rarement d'autres termitières appelées hirgasl, des Odontotermes erratius ou des Trinervitermes qui prospèrent sous certains arbres et épouseraient la «forme d'une tombe». Comme pour azlazlang, termitière sans orifice de sortie, l'essaimage des ailés a lieu à différents moments de la nuit ou après une journée de pluie ( abess) ou parfois lors d'un changement de direction du vent du nord-est vers le sud.

11 Certains chasseurs de termites construisent des enveloppements de chaume proches de la paroi de la termitière pour dissuader l'envol des imagos ou pour qu'il soit reporté à plus tard selon la volonté du " propriétaire ». On pouvait ainsi atteindre la fin du mois d'août, les imagos ralentissant leur processus de croissance. L'arrivée de ces termites au moment de la soudure serait la bienvenue. Quant à leur prix, il doublerait. Dans le dictionnaire Guiziga (du sud)-Français (Jaouen et al 1998: 81) ${ }^{4}$, on peut lire: «magwac/mabang: termitière enveloppée contre la pluie, on enlève l'enveloppe au temps voulu, pour que la pluie la mouille afin que les termites sortent ». Le contrôle volontariste de l'essaimage des termitières est une assertion unanime des informateurs, qui signalent aussi que la pratique était plus courante dans le passé. Pour ma part je n'ai pas été témoin de ces reports programmés d'essaimage. Le procédé des termitières appareillées semble fort ancien si l'on fait l'hypothèse - partagée par nos informateurs - de lui attribuer les tessons d'anciennes grosses jarres à eaux trouvées sur des planosols éloignés des sites 
habités. Ces réserves d'eau devaient déjà servir à alimenter, le moment venu, les rigoles creusées autour des termitières ${ }^{5}$.

Les habillages de termitières concernent la région de Massakal, Mogoudi, Kaliao et Djapay (au sud du mayo Tchanaga). Le procédé renvoie à la culture mowo dont les officiants rituels prétendaient commander au monde et plus précisément à celui des insectes (Seignobos 2015). Au nord de Maroua, à Gayak et Kosséwa, on n'habille pas les termitières. Les veye'd s'envolent pour retomber sur des sols incultes (harde) qui reflètent la lumière lunaire ou même les rivières en eau. Là, ils perdent leurs ailes et se réfugient sous des tiges de sorgho ou des fèces de bétail. Le lendemain on trouve les termites accouplés essayant d'échapper au soleil, à la recherche de la moindre cavité où fonder leur colonie.

Dans la région on ne consomme pas les ouvriers et soldats termites aptères mais seuls les adultes sexués ailés peuvent être grillés. Les termites sont collectés dans des sacs avant d'être lavés, puis séchés au soleil sur des nattes. Ils subiront ensuite un vannage pour ôter ailes et sable. On veille à ne pas mélanger les espèces. Ces kurbanaani (= ailés en fflde) commercialisés sous ce nom sont vendus à la cuvette (daaro) ou à la tasse (agoda, d'une capacité d'un kilogramme de sorgho) qui sert à mesurer le mil. Le mois de juin reste à Maroua celui des marchés de termites, la tasse trouve preneur entre 500 et 1000 francs CFA. La production par termitière s'évalue à un ou deux sacs de termites. Pour ilimi'd on peut espérer en moyenne une vingtaine de tasses à 400 francs, soit au total 8000 francs et, pour veye'd, une quinzaine de tasses à 550 francs; ainsi les différentes termitières offriraient des rapports équivalents.

Les termites sont grillés dans des sortes de poêlons ou des fonds de canaris. On les sale au dernier moment, avant de les commercialiser ou de les consommer. On peut aussi vendre des boulettes (goplo en giziga) de termites écrasés avec des condiments.

L'exploitation des termitières reste une activité propre aux piémonts des monts Mandara. On ne la retrouve ni en plaine, ni en montagne pourtant proche. Sur les monts Mandara on assigne aux termitières - moins nombreuses - d'autres services, avant tout agronomiques. Dans les fonds des talwegs, le potentiel de fertilité d'une parcelle se mesure en comptant le nombre de Faidherbia, de fourmilières et de termitières. On assiste à une forme de concurrence entre les hommes et les femmes; les premiers cherchent à attirer et à faire se développer certaines termitières sur leurs champs alors que les femmes veulent exploiter le contenu des termitières pour leur volaille en y disposant de vieilles poteries hâtant parfois le départ de la colonie ${ }^{6}$. Cette rivalité s'exprime à travers des joutes chantées entre les hommes et les femmes chez les Mofu nord (à Douroum et Douvangar), au cours des travaux en commun sur les champs.

Dès sa désertion par la colonie, une termitière va accueillir une infinité d'occupants opportunistes (Dounias 2016: 286). Ces hôtes comptent par ailleurs parmi les plus intéressés par la consommation de termites : mangoustes, écureuils fouisseurs, rats de Gambie, hérissons, varans, pangolins, et l'on rencontre aussi civettes, genettes, ratels, chats sauvages, renards des sables... Certaines termitières n'abritent que quelques pensionnaires alors que d'autres, par leur situation et leur architecture interne qui développe en profondeur un impressionnant réseau de galeries et de loges, relèvent de l'arche de Noé. 
Figure 3 : Chasse aux termites, village de Mogoudi

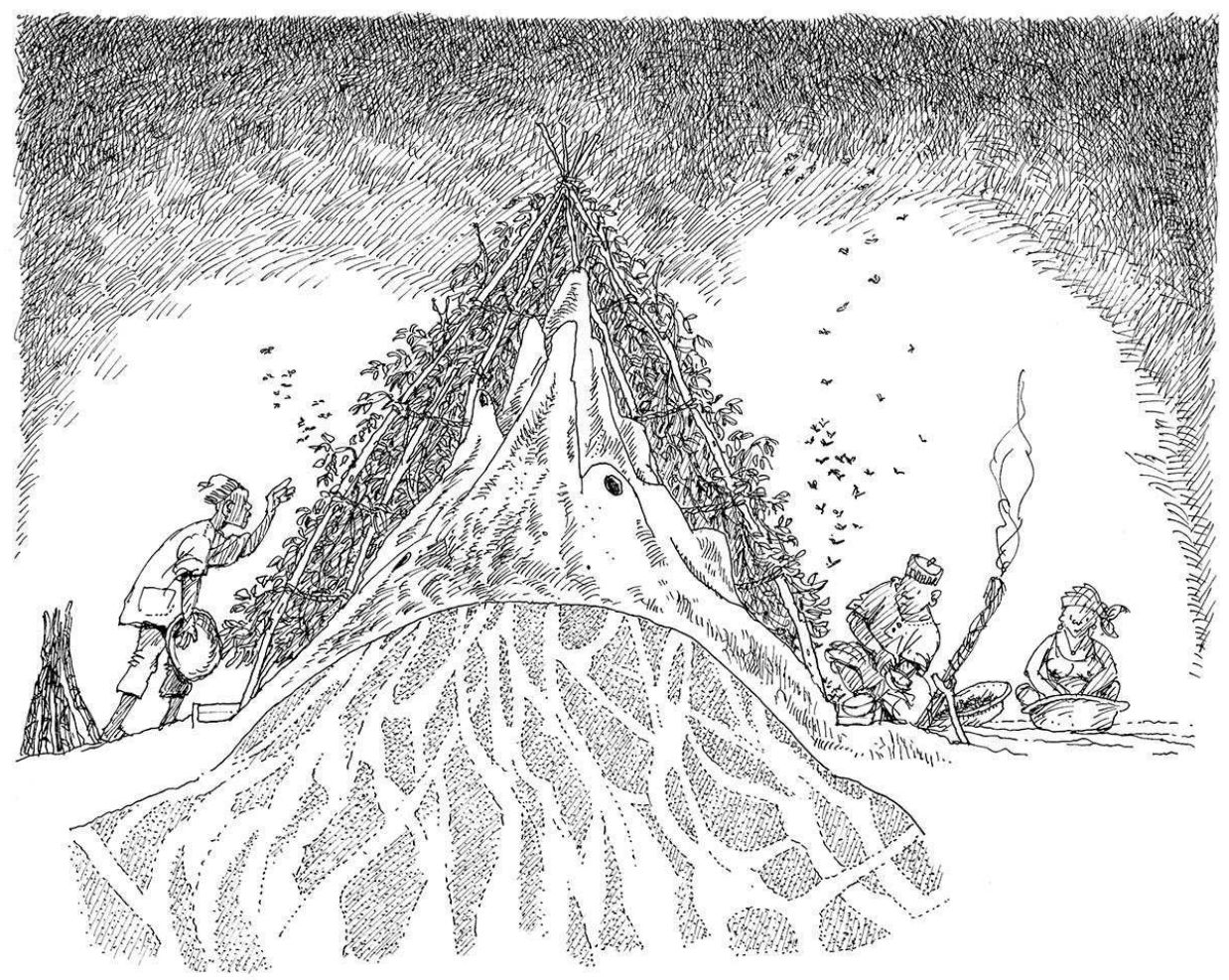

Dessin C. Seignobos

\section{Les animaux des termitières mortes, un exemple d'éthologie giziga}

17 Les termitières désertées par leur colonie s'identifient de maintes façons. Par exemple un écureuil fouisseur qui, poursuivi, pénètre dans une termitière et n'en ressort pas serait un bon indicateur d'absence de termites. Les termitières vivantes ne sauraient longtemps cacher un animal, rapidement incommodé par ses gardiens termites. Néanmoins, seul parfois, l'appareil aérien a été abandonné par les termites qui demeurent alors dans les parties basses et accueillent ainsi des logeurs «à l'étage ». Ces derniers ne seront que temporairement importunés par les attaques de soldats qui accompagnent et protègent l'essaimage.

Chacune des espèces hôtes aménage de façon quasi identique sa loge et ses accès. Elles peuvent ou non, au cours de l'année, investir d'autres sites refuges où elles mettent parfois bas durant la saison des pluies. D'autres, en revanche, investissent les termitières plutôt pendant la saison humide.

\section{Le monde des souris et des rats, locataire occasionnel des termitières}

Les « souris » - qui sont souvent des rats - peuvent se réfugier dans les termitières. Elles ne sont guère outillées pour aménager et encore moins creuser les termitières épigées. On les retrouve, en revanche, dans les termitières basses, azlazlang, constructions plus 
meubles, faciles à forer, avec une préférence pour celles à proximité de végétations buissonnantes. Nous ne mentionnerons que certains de ces petits rongeurs occasionnels.

Figure 4 : Les rongeurs des termitières

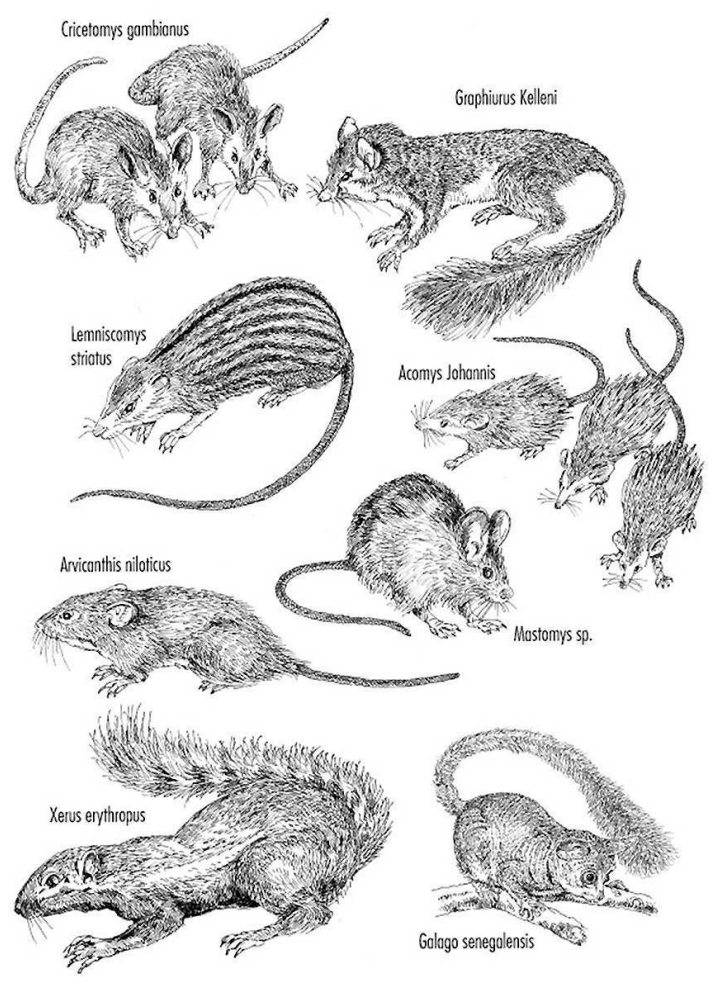

Dessin C. Seignobos (Granjon \& Duplantier 2009)

Moco'd/motso'd $\mathbf{d}^{7}$, le rat du Nil ou rat roussard (Arvicanthis niloticus), est appelé « souris » par les Giziga. Ce rongeur diurne, très ubiquiste, se nourrit généralement de graines et de racines, mais pendant la saison des pluies il est volontiers insectivore. Lors de la saison sèche, il peut adopter un comportement arboricole, en particulier sur les balanites. Il creuse des terriers ni très longs ni très profonds. Grégaires les moco'd vivent nombreux dans leurs terriers. Les bouches des terriers sont toujours rapprochées. Ils aménagent des galeries de secours vers des sorties échappatoires maintenues fermées (munndul) ${ }^{8}$. Ils ne cohabitent pas avec d'autres « souris », mais peuvent accepter la présence du hérisson et du varan. Toutefois l'arrivée d'un serpent chasse les moco'd, avertis par son « odeur » ils peuvent déménager en une journée, et logiquement leur présence dans une termitière signifie l'absence de reptiles. Moco'd occupe l'étage intermédiaire d'une termitière et sort deux fois par jour, matin et soir, pour aller chercher sa nourriture dans les zones herbacées et les champs de mil. On le rencontre rarement dans le mitan du jour. Il se réfugie dans la journée sous les clôtures d'épineux et les buissonnements de riya (Acacia ataxacantha) ou encore sous des tas de chaume. Leurs va-et-vient tracent des chemins très visibles. Les moco'd sortent et rentrent en suivant ces couloirs l'un après l'autre, ils risquent alors de mauvaises rencontres avec le python ou des couleuvres qui les attendent sur leur passage. Le python tromperait les moco'd en imitant les petits cris et sifflements des rongeurs lorsqu'ils cherchent leur pitance et rameutent les autres. Les femelles mettent bas dans les termitières pendant la saison des pluies, mais, pour la portée de saison sèche elles profitent des fentes de retrait des argiles, voire de celles des 
mares asséchées. Dans ces fentes (dalak ou boli en fflde) les femelles mettent bas dans des nids garnis d'herbes (Bulbostylis sp.), justement appelées « herbes des souris » (hu'do doombi en fflde).

Meseseng, Mastomys kollmanspergeri, un autre petit rat, se rencontre un peu partout. Il fait ses terriers dans les tas d'ordures anciens (kitikil) au sol meuble ou il choisit azlazlang, termitière fermée, mais facile à aménager. C'est là que la femelle met bas ses nombreuses portées. On gratifie meseseng d'un odorat plus fin que tous les autres rongeurs. Aussi, chez les Giziga, on dit de quelqu'un qui pressent les choses qu'il a l'odorat d'un meseseng. Seuls les enfants le chassent en raison de sa petite taille.

Mokwolay (mocokru en fflde), le rat rayé (Lemniscomys striatus), pour les Giziga, «la souris arc-en-ciel » (kwolay) en raison de l'alternance de bandes médianes brunes et claires latérales et longitudinales. Il ne creuse pas de terrier mais récupère ceux des autres. Les Giziga craignent cette "souris arc-en-ciel», comme ils redoutent les kwolay, nom donné aux pierres qui arrêtent la pluie. Les serpents ne l'attaqueraient pas, mieux, pour fuir elle se transformerait en serpent sur le modèle de sa longue queue si semblable à celle d'un reptile. On interdit généralement aux enfants de la chasser.

Muluwa'd ou mapparaw ou encore atsang (dabuuru en fflde, le petit loir, Graphiurus kelleni). Au delà de son poil de couleur grise et d'aspect laineux, les Giziga remarquent surtout sa queue touffue et en panache. Nocturne, à la fois végétarien et insectivore, il passe par des orifices plus grands que sa taille et qu'il laisse très propres, mais on distingue la marque de ses griffes, ses traces seraient légèrement plus grosses que celles du chat sauvage. Muluwa'd peut éventuellement vivre dans la même galerie que l'écureuil fouisseur, Galago senegalensis, et le varan. La femelle met bas dans les termitières protégées par un embuissonnement de riya. C'est surtout un arboricole des gros arbres, Ficus spp. et tamariniers. Il parviendrait aussi à pénétrer dans les enclos des hommes et même dans les toitures des cases. Il se voit ainsi accusé de tous les incendies d'habitation non élucidés, le poil de sa queue prendrait feu près d'un foyer et avec ce brandon il propagerait l'incendie de case en case.

Munduwang (ndoonbala ou doomru woyla en fflde), le rat de Gambie (Cricetomys gambianus), dit « rat voleur ». Ce gros rongeur de couleur sombre, à longue queue, creuse des terriers profonds dans les termitières, mais aussi dans les concessions des villageois. Dans la termitière il se place sous l'étage de l'écureuil fouisseur et il aménage son terrier de plusieurs sorties possibles et de nombreux carrefours. Il se réfugie aussi dans les troncs d'arbre, sous les bottes de paille et de tiges de mil. Ce nocturne est accusé d'être un grand pilleur de champs d'arachides et il serait assez hardi pour dévorer tout ce qui est entreposé pour séchage sur les auvents : mil, pois de terre, niébé, courges, restes de boule de mil. À ses heures le rat de Gambie est insectivore. Sur les termitières azlazlang il est, après le départ des termites, le premier avec l'écureuil fouisseur à venir consommer les champignons blancs (Termitomyces spp.). Toutefois la particularité du rat de Gambie est d'aménager une loge conséquente pour stocker de la nourriture : fruits, graines, petits tubercules... qu'il transporte dans ses bas joues en poches. "Il se comporte un peu en propriétaire ", ce qui gênerait les autres pensionnaires de la termitière qui, comme l'écureuil, risquent d'aller creuser leurs terriers ailleurs.

Meferew, Acomys johannis, est « une petite souris qui a des poils un peu longs, brillants et presque épineux à l'arrière, sans toutefois blesser le chasseur qui l'attrape ». Elle vit plutôt dans des amas de pierres, sous la paille, des paquets de tiges de sorgho et, parfois 
aussi, elle se réfugie dans des termitières. Nocturne, elle a la réputation de ravager les parcelles de pois de terre et d'arachides. Les Giziga la créditent de recéler plus de graisse que les autres.

\section{Les locataires permanents des termitières}

26 Ayew (jiire en fflde), l'écureuil fouisseur (Xerus erythropus), préfère creuser ses propres terriers dans les termitières. Il profite de la saison humide et du sol meuble pour forer de nouvelles galeries. Grâce à de solides griffes il expulse la terre avec ses pattes arrière. Il se ménage une loge centrale d'où partent deux à trois galeries au plus. Certaines de ces galeries basses seront ensuite récupérées par le hérisson, puis agrandies par le varan avec ou non un reptile colocataire. Ses ouvertures sont généralement les plus élevées de la termitière au-dessus de 1,60 $\mathrm{m}$ et seuls les grimpeurs: écureuils fouisseurs, varans, civettes, chats sauvages, peuvent s'y hisser. Toutefois s'ils entrent par le haut ils ne gitent pas forcément à cet étage. Le cobra cracheur peut être une menace pour ayew mais il craint le varan, car s'il y a combat les deux peuvent en mourir. Aussi l'écureuil chercherait-il systématiquement la proximité du varan. Les chasseurs giziga affirment que «le varan sauve l'écureuil ».

27 L'écureuil fouisseur n'a pas d'horaire pour quitter ou intégrer sa cache. Il sort et rentre à tout moment, mais le jour, jamais la nuit. Il s'abstient par grand vent car il ne peut repérer les bruits des pas de l'homme ou la présence des chiens. Sa nourriture est variée : grains, racines, bulbes, fruits tombés au sol et aussi insectes. Durant la saison des pluies l'écureuil fouisseur va mettre bas dans les troncs d'arbres, puis revient à la termitière. Il ne s'éloigne jamais de la termitière d'où le proverbe bien connu : « l'écureuil affirme bien sûr croire en Dieu, mais juge plus prudent de ne jamais trop s'éloigner de sa termitière ». L'écureuil porte chance: en voir un au début de la journée demeure un bon présage, encore faut-il garder pour soi cette rencontre. Chez les Giziga, comme chez leurs voisins, l'animal reste le principal acteur positif de très nombreux fabliaux.

Nocturne, Dalmavay (joldu en fflde, le petit lémurien, Galago senegalensis) sort de la termitière pour se réfugier dans les arbres, avec une préférence pour les balanites ( Balanites aegyptiaca). L'entrée du terrier est propre, seuls quelques poils peuvent le trahir. Ce lémurien aurait la particularité de résider avec la plupart des autres animaux et sur plusieurs étages.

Tsatlak/tsazlak (kiyal ou macamaawal en fflde, la civette, Civettictis civetta) est un viverridé nocturne qui rentre à l'aube. Cette civette partage le même étage que l'écureuil. Elle creuse son terrier et aménage une grande loge pour mettre bas ses petits. Sa portée une fois là, la civette va alors chercher à chasser les autres pensionnaires de la termitière. L'odeur forte qu'elle dégage de ses glandes à musc peut être dissuasive pour d'autres occupants.

Maavay (kuyrawal en fflde, la mangouste rouge, Herpestes sanguinea), peut être également présente à cet étage.

31 Magamak nguu 'boo (kosebe en fflde) désigne le chat sauvage (Felis sylvestris lybica). Les contes ou les fables, plutôt chez les islamisés, en font un animal féroce qui, dans une version amplifiée, devient un objet de terreur pour toute une région. Il se révèle un locataire difficile, voire exclusif de la termitière, car il en chasse peu à peu les autres 
animaux. S'il y a affrontement avec un serpent, ses griffes redoutables et son agilité affolent le reptile, qu'il finit par dévorer.

Biika (doldoln'de en fflde, le renard pâle, Vulpes pallidus), creuse des terriers très profonds et les aménage de multiples sorties. Son gîte abandonné est récupéré par le python. Ce renard des sables a la réputation d'être difficile à tuer et serait porteur, à la façon des hommes, d'un nom personnel. Il est censé arrêter la pluie et peut «fermer la brousse » aux chasseurs, ce dont nous aurons à reparler.

Yeendu en fflde, l'oryctérope (Orycteropus afer) est appelé « kangourou » dans le français local (Seignobos \& Tourneux 2002). Il recherche les plus grosses termitières, mais surtout les plus anciennes, celles qui ont subi une inondation ayant agrandi et élargi galeries et ouvertures. Yeendu aménage une loge vaste pour mettre bas ses petits. Sa présence est facilement repérable, surtout sur les termitières des grandes plaines herbeuses, les yayrés, avec la marque de ses griffes. Sur les piémonts des Mandara il est devenu rare. Image du Dieu Seth d'Égypte on se souvient de son aspect étrange et effrayant dans les rencontres nocturnes, mais on vante encore la qualité de sa chair.

34 Le ratel, Mellivora capensis, est un petit blaireau sombre, au dos plus clair. Il possède de grandes griffes, il est très présent dans les termitières, c'est aussi un nocturne.

\section{Colocataires compatibles, animaux à piquants, varans et serpents}

Cecem (tsemtseme, samnalde, camnale ou gedegel ladde en fflde, Atelerix erinaceus) est un hérisson vivant souvent en colonies dans les termitières. Pendant la saison des pluies il sort mettre bas dans le creux des arbres ou dans les grosses fentes des terres à karal (argile à montmorillonite à fentes de retrait), sous les herbes rampantes. Il construit des nids un peu comme ceux des rats, il met également bas dans les galeries de vieux terriers de termitières qu'il a récupérés. Les hérissons choisissent les entrées les plus basses des termitières. Ils peuvent cohabiter avec des serpents. Pour protéger sa progéniture, le hérisson bloque la galerie en faisant la boule devant le serpent qui, alors, recule. Il reste cependant vulnérable devant certains rapaces comme le rawrawa (non identifié) 9 .

Didir (sanalde en fflde, le porc-épic, Hystrix cristata), dont dos et flancs sont couverts d'épines érectiles, annelées, noires et blanches, combinant des longues et souples et des courtes et raides, habite tous les terriers d'une certaine dimension, y compris ceux des termitières. Nocturne il consomme racines, bulbes, fruits, insectes. Sa chair est recherchée tout comme ses longs piquants qui se rigidifient après la mort de l'animal. Avec l'oryctérope, le porc-épic fait partie des animaux chtoniens qui réclamaient un traitement particulier à leur mort. 


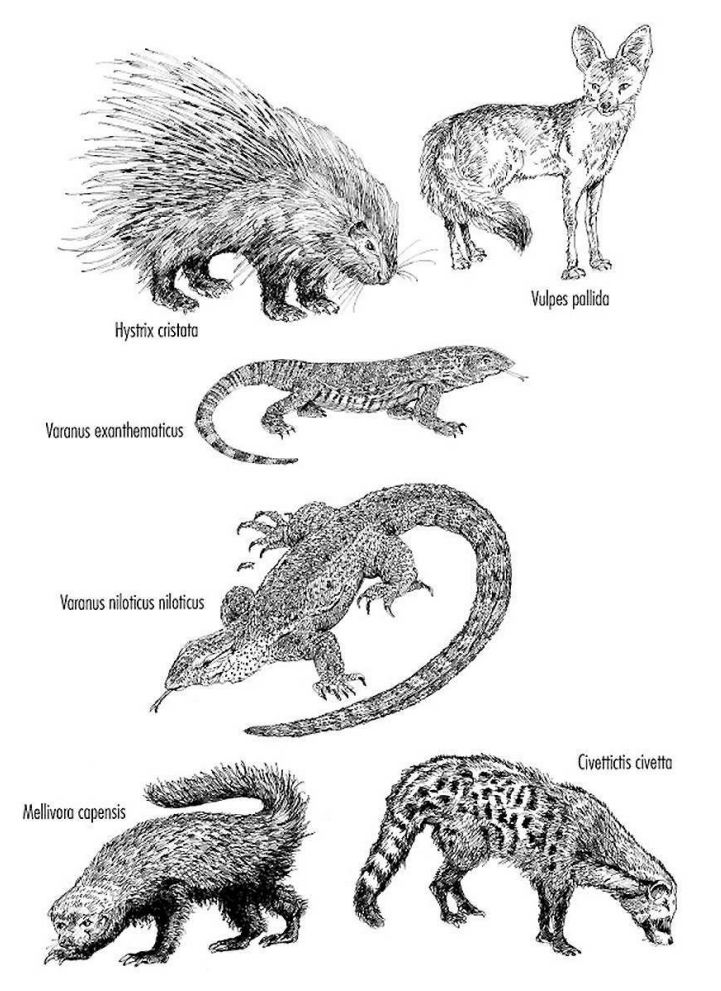

Dessin C. Seignobos

Mawbrun, mon'burong (huutooru ou lawnyi en fflde, le varan terrestre, Varanus exanthematicus), résiderait six mois dans la termitière et l'autre partie de l'année, durant la saison des pluies, il choisirait de vivre sous les arbres. Il sort de la termitière à midi, lorsque le soleil est haut et il rentre avant la nuit. Il quittera la fraicheur de la termitière après les premières grosses pluies, lorsque la brousse commence à être saturée d'eau et où partout l'explosion végétale s'accompagne de myriades d'insectes.

Les varans changent rarement de termitières et empruntent toujours l'ouverture du même terrier où s'observent les traces de leurs griffes. Ils pondent leurs œufs en août, à la fin de la récolte des légumineuses niébés à l'extérieur de la termitière. Après éclosion, les petits varans cherchent leur nourriture dans les herbes. Le varan de terre appelle la chance : après sa rencontre on effectuera un bon voyage, on réussira ses ventes ou ses achats sur le marché. Alors que je traversais une brousse dans le terroir de Djébé, en pleine saison des pluies (1992), arrivé sous un arbre, un de ceux qui m'accompagnait, un Giziga islamisé, se précipite à mes pieds et saisit un varan caché dans les herbes. Il le maintient par la nuque et se met à lécher la longue langue cylindrique du varan avant de le relâcher, extasié. Et tout mon entourage de le féliciter pour l'année heureuse qui s'ouvre devant lui.

Duwel (el'eldu en fflde, le varan d'eau ou varan du Nil, Varanus niloticus niloticus), plus grand que le précédent, se reconnaît à sa couleur verdâtre et à sa longue queue avec laquelle il fouette ses adversaires y compris les serpents. Sa morsure est redoutée. Il demeure dans des termitières ou des arbres proches des rivières ou des mares. Comme le varan de terre il partage les mêmes galeries que le cobra ou le python. Contrairement à mawbrun, duwel provoque la malchance et il convient de s'en prémunir. 
40 À l'arrivée des serpents, kwa, nom générique en giziga, toute la gente des souris et des rats cherche à déménager de la termitière. En revanche, les varans, les hérissons et même l'écureuil peuvent utiliser la même entrée que les serpents. À l'intérieur, des bifurcations dirigent chacun vers ses appartements. Toutefois les serpents gitent dans les étages inférieurs des termitières. Les plus gros y pénètrent par l'arrière, par la queue et empruntent toujours la même galerie.

41 Govong (culanndi en fflde, le cobra cracheur à cou noir, Naja nigricollis nigricollis), peut, comme le varan, sortir au milieu du jour. Au retour le varan précède le cobra, qui rentre à la nuit tombée. Le cobra est aussi un nocturne qui affectionne les déplacements sous les acacias Acacia ataxacantha et revient de grand matin, l'ouverture de son terrier est parfaitement lissée. Il dépose ses œufs dans la termitière. Il représente souvent le plus vieux pensionnaire et peut résider une quinzaine d'années à la même place.

Tizlang, tunjang (modaari ou mo'da baaliiri ou encore mala'bla'beeri en fflde, le python de Seba, Python sebae), appelé «boa » dans le français local, intègre également la termitière par la queue pour rejoindre sa loge et y effectuer ses longues digestions. Il dispose généralement du terrier le plus profond.

Cette liste d'animaux gîtant dans les termitières n'est bien sûr pas limitative. Ce que le chasseur cherche à connaître ce sont les parcours des gibiers vers les termitières et les horaires de sorties et d'entrées, pour les diurnes comme pour les nocturnes. Cela conduit aussi les chasseurs à acquérir une connaissance fine de la cohabitation d'animaux partageant la même termitière et de connaître tous les conflits potentiels entre ces colocataires, sources d'inspiration inépuisables pour narrer histoires et fables et animer les soirées de saison sèche. Parmi ces chasseurs informateurs, Hamidou Angokay, de Djébé, s'est montré le plus passionné et le plus disert. On aurait pu enregistrer les histoires de chasse d'Hamidou comme des sortes de proto-contes giziga.

\section{La chasse dans les termitières mortes : la pratique}

44 C'est un mode de chasse qui intéresse plus largement les terriers, qu'ils soient creusés dans les berges des rivières, les dunes ou encore les troncs d'arbres. Il s'agit de débusquer le gibier par enfumage, creusement, inondation et autres "tisonnages». Chasser dans une termitière ne se décide qu'après un long suivi, parfois sur deux années. Après avoir supputé l'infrastructure de la termitière, la disposition de certaines galeries, des loges, les chasseurs évaluent le taux de remplissage et l'effort à consentir pour en extraire le gibier. Aussi procèdent-ils régulièrement à ces relevés de traces sur les abords des termitières, celles des griffes de varan, plus fortes encore celles du ratel, celles, incomparables, de l'oryctérope, mais encore toutes celles, minuscules, des petits rongeurs. Ils relèvent aussi les traces laissées par les serpents et celles dissemblables entre les queues des deux espèces de varan. Pour le python également, c'est par le mouvement final de la queue, que l'on décèle assez facilement son passage. Les fèces font naturellement partie de l'expertise. Les chasseurs observent les va-et-vient, leur rythme et cherchent à repérer l'existence de nichées. Ils écoutent, à partir des ouvertures, les bruits de l'intérieur. Termitières vives ou mortes, elles sont fortement auscultées à l'oreille.

Cette chasse se pratique par petits groupes avec, à la tête, le " spécialiste ", mu sina go'd, qui assure le diagnostic et décide du moment de la chasse. Il est flanqué d'un à trois adjoints, miji gabal, «le porteur de sac», ou encore suuka (en fflde), «le jeune» (ou 
l'apprenti). Les préparatifs rituels pour la saison de chasse ou pour une campagne particulière sont légion. Signalons que les chasseurs consomment des oignons frais et les frottent sur leur corps, l'odeur en éloignant les serpents. Ils consomment encore du natron (kilbu) ${ }^{10}$ contre le venin, ce qui affaiblirait la toxicité lors de la morsure. Dans les chasses au terrier, le danger, bien réel, provient des serpents.

Les chiens sont le plus souvent présents. Ils signalent le gibier qui, en ralliant la termitière, a emprunté telle ouverture mais, surtout, ils poursuivent celui qui a échappé à l'enfumage ou à la capture. Les chiots sont entrainés lors de chasses particulières celle qui s'intéresse à la plus rapide des gerbilles, Gerbilliscus gambianus. On leur fait régulièrement prendre des «médecines de chasse » (matay). Pour stimuler l'odorat, ce sera des bulbes du genre Crinum écrasés avec de la chair de musaraigne. Pour qu'il saisisse bien le gibier, l'ingrédient de base consommé est le soldat termite, le remède s'appelle d'ailleurs manjara matay. Il utilisera ses crocs comme le fait le soldat termite avec ses mandibules et il ne lâchera pas sa proie.

Les chasseurs de termitières disposent des mêmes armes que pour les chasses collectives de village (bula keri) : la hache à pic ou crochet (siikataare en fflde) pour achever le gibier, des javelines à barbules et harpon (swar) ou barbules et pointe (juwet et jaaral en fflde). Des bâtons de jet (tokom) dont les plus courants possèdent l'extrémité distale en boule ou en forme de marteau. Les Giziga Kaliao et Djébé en utilisent un particulier à deux pics dit cekolek. Des bâtons de jet droit, plus allongés (ganday) portent, pour certains, une extrémité crossée ${ }^{11}$. Avant l'ouverture de la chasse tous sont enduits de "remèdes ", on y écrase des becs, des ergots de coqs, des toiles d'araignées...; ici la théorie des signatures explicites joue à plein. On n'oublie pas, bien sûr, le sac de cuir njidka qui identifie le chasseur. 


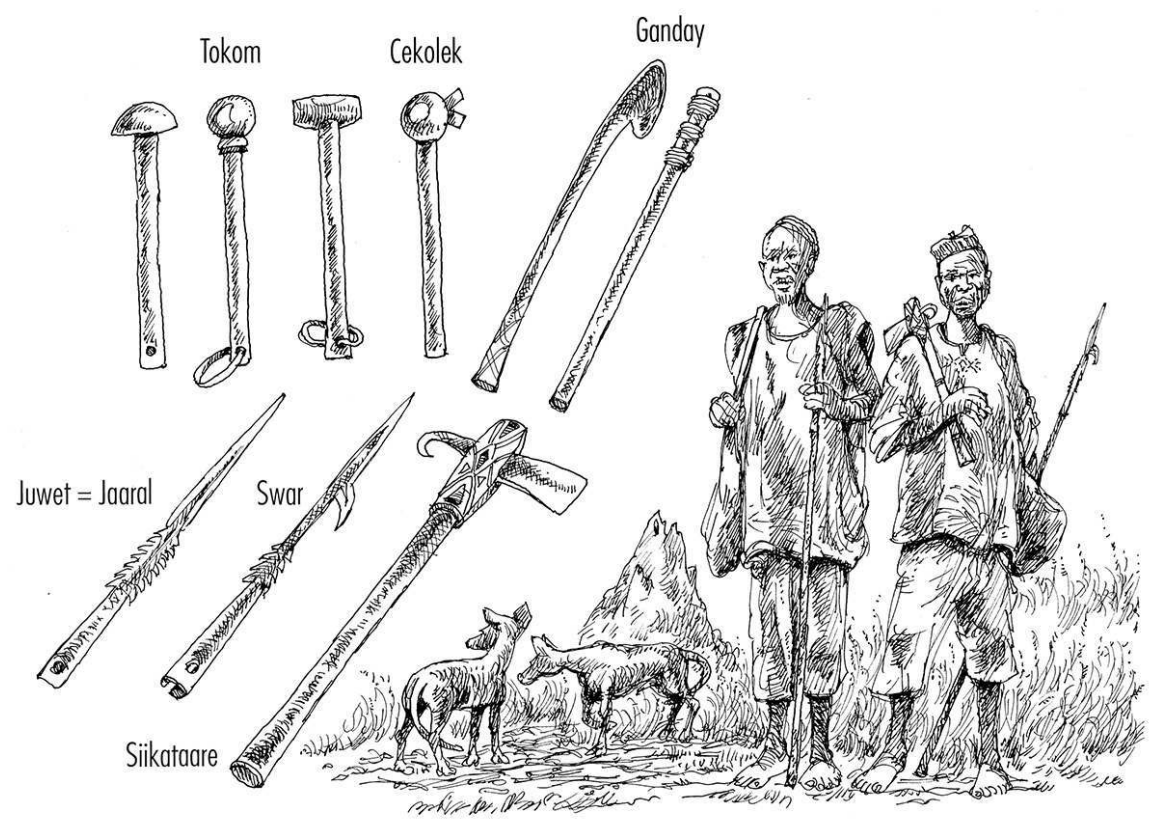

Chasseurs de termitières

Dessin C. Seignobos

Les chasseurs de termitière participent aussi aux chasses collectives de leurs villages respectifs dont ils partagent tous les codes, comme les cris poussés par celui qui a touché de son arme un animal à poil à la tête: to'o, à la patte avant: pasl, à un membre postérieur : nduuk...

\section{L'enfumage}

On n'enfume pas toutes les termitières. À la différence de veye'd, celle d'azlazlang est généralement creusée car elle est censée abriter des esprits (shetene) puissants capables d'entraîner quelques folies difficiles à soigner. On y retrouve pourtant les mêmes locataires. L'enfumage de la termitière se déroule, comme celui des arbres creux, le matin ou en fin de journée avant le coucher du soleil. On ne le fait jamais au milieu du jour, c'est en effet le moment ou certains esprits prennent des formes animales : le varan d'eau, la civette ou encore le cobra...

L'enfumage est théoriquement une technique simple. Les feuillages sont prélevés dans les environs et il s'agit souvent d'un mélange intégrant des feuilles fraiches de Piliostigma reticulatum. On s'abstient d'enfumer avec des branches de tamarinier et celles d'arbres nourriciers Ficus gnaphalocarpa ou Ficus platyphylla. Tous les orifices de la termitière doivent être bouchés par des paquets d'épines à l'exception d'un seul par lequel la fumée va pénétrer. On y verse les braises d'un feu allumé à proximité. Sur ces braises on jette, au dernier moment, du piment (citta masaaru en fflde), un Capsicum sp. très fort qui accentue l'effet de la fumée, qui pique à la fois les yeux et le museau des animaux. On peut également répandre sur les braises du tabac mais c'est plus coûteux. L'effet du tabac 
« enivrerait » l'animal et à la sortie il titube, ne sait où aller et se laisse facilement attraper par les chiens, spectacle dont s'amusent les chasseurs en particulier avec le petit rongeur, Graphiurus kelleni, et les hérissons.

51 On use alors d'un soufflet, le plus souvent en branches de feuillus pour faire pénétrer la fumée avant de fermer prestement l'orifice. La fumée se répand, indisposant tous les pensionnaires de la termitière. Ils s'agitent alors et se dépensent sans compter dans le labyrinthe de la termitière. On rouvre le trou que l'on recouvre d'une pièce de pagne bleu élimé qui ne laisse pas passer la fumée mais donne un peu de jour. Le "spécialiste» s'allonge sur la termitière tout en maintenant avec ses deux mains écartées le tissu obturant l'ouverture, il écoute et traduit les bruits qui parviennent des galeries.

Les animaux sont classés par rapport à leur sortie de l'enfumage. L'écureuil fouisseur, très sensible à la fumée, sort le premier. Il dispose, rappelons-le, d'un terrier quasi individuel et peu ramifié situé à l'étage supérieur. Les varans suivent et s'ils sont les premiers, c'est que la termitière n'abrite pas d'écureuils. Viennent alors des locataires irréguliers occupant des loges plus souterraines : ratels, porcs épics et d'autres qui créent souvent la surprise. Les serpents signent les dernières sorties, la termitière est alors vide ou ne restent que les animaux qui sont morts. Lors des enfumages il convient de maintenir les chiens à distance car certains animaux préfèrent la mort par asphyxie plutôt qu'affronter les chiens. L'animal ne sait comment résoudre le dilemme de son enfermement dans la termitière, il souffre de la fumée, se débat et, épuisé, il sort, souvent dans un dernier effort.

L'interprétation des bruits dans la termitière serait essentielle. Elle passe par une grille d'onomatopées répertoriées pour chaque animal que les chasseurs reconnaissent et enseignent à leurs « jeunes ». Toutefois les onomatopées retenues se révèlent parfois très différentes selon les villages comme Kosséwa et Kaliao, par exemple.

Lors des enfumages l'écureuil ferait guduk, guduk, guduk, dans sa lutte contre la fumée et quand il se décide à sortir il émet un cri : krowo wo wo ou kwukwukwu... perceptible par le chasseur. Il peut s'élancer deux à trois fois et rebrousser chemin. L'animal voit le jour à travers le tissu bleu. Il se décide enfin et se précipite. Le tissu est toujours maintenu par l'extérieur et les deux mains se refermeront sur la tête de l'animal.

Lorsqu'ils courent à travers les galeries, les deux espèces de varan émettent des sons qui ne sont pas distinctifs. Ils commencent au fond de la termitière par kurwo wowo wowo avant de le transformer à l'approche de la sortie. Les varans effectueraient trois à quatre va-et-vient avant de se décider.

La civette, sur le même étage que l'écureuil, effectue encore plus de tours, tentant les différents orifices, avant d'admettre que le seul qui reste ouvert serait celui obturé par le tissu. Elle émettrait des k'wa k'wa k'wa avant de foncer.

Le galogo (Galago senegalensis) peut se retrouver sur plusieurs étages et la fumée le fait courir, monter et descendre. Ses allers-retours sont aussi nombreux qu'irréguliers. Il se jette sur le tissu qu'il faut souvent renouveler après son passage.

Devant la fumée, le chat sauvage et le loir, Graphiurus kelleni ne poussent aucun cri. Ils montent et descendent souvent, seuls les frottements de leur corps contre les parois des galeries seraient perceptibles pour le chasseur. Ils préfèrent en général mourir dans la termitière. 

qui, devant le tissu qui voile l'orifice, deviendraient : haa, haa, haa... Il peut descendre et remonter deux à trois fois. Le chasseur aux aguets, toujours allongé près de l'ouverture, l'arrête aussi par la tête avec le tissu et le tire hors de termitière. Son aide l'égorge sans jamais trancher complètement la tête du cobra. Deux porteurs le transportent enroulé autour d'une perche.

60 bruit de grand vent ». Il remonte jusqu'à l'ouverture, mais souvent il ne peut se résoudre à sortir, aussi mourra-t-il dans la termitière et il conviendra de creuser un puits pour le trouver ${ }^{12}$.

\section{Le creusement et l'inondation}

61 On creuse dans le cas où l'enfumage ne donne pas satisfaction. Sur des terriers trop longs la fumée s'arrête en chemin. Les termitières récentes, encore humides, conduisent mal la fumée.

62 Une fois les locataires de la termitière dûment répertoriés on décide alors, selon leur étage et leurs loges pressentis, de boucher toutes les sorties avec des épineux d'acacias et de jujubiers. Les chasseurs creusent des puits au-dessus des bruits entendus. Ils se livreront à des «tisonnages » avec de longs bois frais et souples. Ils feront réagir l'animal que l'on peut alors atteindre et confirmer sa nature, poils, écailles, piquants... Arrivé audessus du boyau où s'est réfugié l'animal le chasseur pique avec sa lance à barbules. Si l'animal a pu se replier plus au fond, il faut recreuser un puits après avoir rebouché les précédents. Le chien joue ici un rôle important car l'animal blessé cherche à bondir et à sortir. Là on lui envoie des bâtons de jet. Le chien, appelé par son nom, est lancé sur le gibier et en retour il a droit aux entrailles. 


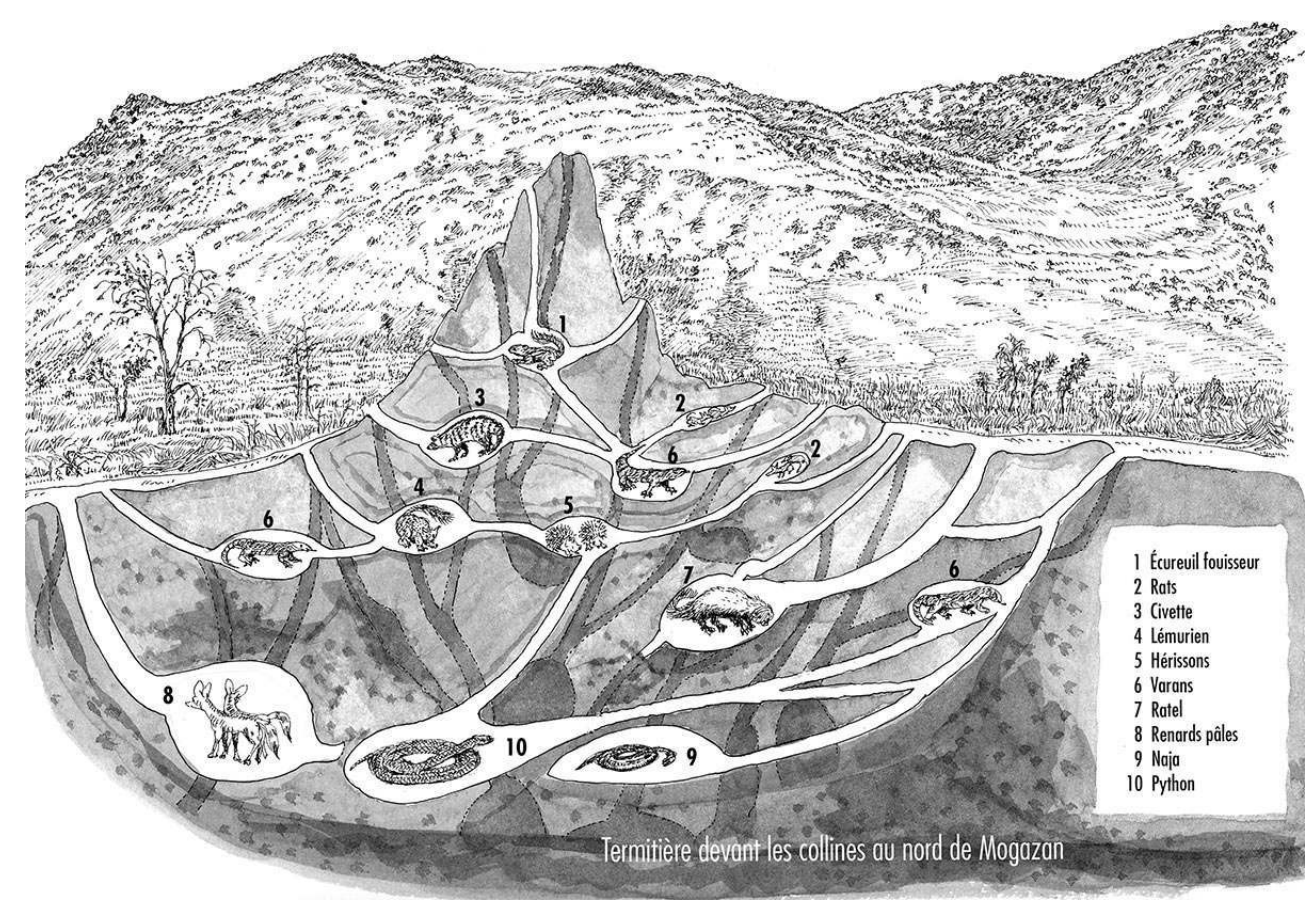

Dessin C. Seignobos

Après une grosse pluie, en juillet, les chasseurs tracent une rigole, parfois un petit canal avec diguettes, pour atteindre les trous les plus bas et ainsi inonder une termitière. L'eau en chasse les habitants, les écureuils encore une fois sortent les premiers, puis les rats et les souris qui se précipitent dans les buissons voisins. Mouillés et transis de froid ils se font aisément prendre. Suivent les porcs épics et les hérissons que le chasseur transperce de sa lance et jette dans un sac. Après avoir beaucoup circulé dans les galeries les varans sortiront les derniers. Au cours de la même saison des pluies les chasseurs pourront revenir sur cette termitière car civettes, genettes, chat sauvages, loirs Graphiurus restés momentanément réfugiés dans les buissonnements voisins s'y seront à nouveau installés.

Les varans relèvent encore d'une chasse spécifique. Ils sont attrapés avec de gros hameçons montés sur une tige de fer souple que l'animal ne peut couper de ses dents. On y accroche une grenouille merlek (Ptychadena sp.) vivante par la patte. Le varan se précipite sur cet appât qui s'agite à la sortie du terrier. L'hameçon prend le varan que l'on remonte et qu'on égorge. Le varan "ne meurt pas vite ", il faut également lui trancher la nuque. Pour le transporter on fait entrer sa queue dans la paroi de la gueule.

65 Les produits de ces chasses ne se retrouvent pas sur tous sur les marchés. Fort peu de serpents ne se consomment pas, depuis la grosse couleuvre azawa (azawaru en fflde) jusqu'au muludede (ffde), Psammophis sibilans, une couleuvre très fine à lignes marron sur fond jaune que l'on laisse près des habitations où elle capture de petits rongeurs. Les chairs du cobra et du python sont recherchées. Le python constitue souvent le plus gros volume de viande de la termitière. Toutefois seule sa graisse est réellement commercialisée. La chair des serpents se partage entre apparentés et voisins.

66 Les gibiers comme les rongeurs Mastomys kollmanspergeri, Lemnicomys striatus, Acomys johannis n'arrivent jamais sur les marchés. Le rat de Gambie, vendu entre 300 et 1000 
francs CFA à Maroua en 2010, n'est commercialisé que pendant la saison sèche car sa viande tourne rapidement avec l'humidité de la période pluviale. Le rat du Nil était la même année à 50 francs pièce et la seule gerbille, Gerbilliscus kempi, était vendue à 25 francs. De loin le plus commun des gibiers de termitière mis à la vente est l'écureuil, de 500 à 700 francs dans les marchés villageois et son prix peut atteindre 1200 francs à Maroua. Les recettes de cette chasse servent des formes de convivialité dans les cabarets à bière de mil, à se procurer du tabac, des noix de cola, du soya (viande de bœuf grillée) sur les marchés, et de menues emplettes.

Il existe un commerce parallèle caché, toutefois plus lucratif, concernant des sousproduits de ces chasses, sous la forme d'os, de dents, de peaux, de viscères à incorporer dans maints talismans. Par exemple les longues épines du porc-épic alimentent dans les montagnes un commerce de protections occultes. On les rencontre fichées à l'entrée des habitations, près de l'autel où se déroulent offrandes et libations. La liste en serait bien trop longue. La peau des petits carnassiers a longtemps fourni la sacoche où l'homme remisait argent, tabac, briquet et noix de cola, comme celle de la civette ou mieux encore de la genette tigrine (Genetta tigrina), dabuuru en fflde.

\section{Les chasseurs de termitière au bas de la hiérarchie de la chasse?}

68 Il existe plusieurs classifications des modes de chasse (Seignobos 2011: 37), la plus courante chez les Peuls, celle qui fait école est la suivante :

- le gawaangal, chasse à l'arc et flèches empoisonnées pratiquée aujourd'hui avec des fusils artisanaux, sans chien, appartient aux musulmans se prévalant de connaissances ésotériques de la brousse ;

- loohngal, de loohaago, «chasser » au sens large, inclut des sous-catégories comme les chasseurs de termitières, en fait des terriers, musulmans et païens ;

- diiwgal, de diwgo, «courir en sautant » est proprement réservée à la chasse collective avec chiens, à la lance et au bâton de jet, également à la chasse à courre, le mot est réservé aux non musulmans car « ce sont leurs chasses ";

- jomngal (on parle plutôt de jomnoowo), de jomnugo, prendre au piège. Les piégeurs sont traités par les gaw (chasseurs professionnels) de " voleurs de brousse » (guujo ladde ). Les pièges (tekkere) sont principalement des pièges radiaires en éclats de bambou ou de tiges de sorgho reliés à un billot de bois, le tout appelé tongolongol ${ }^{13}$.

Les chasseurs de termitière giziga interviennent dans leurs terroirs en marge des parcellaires, sur des sols incultes ou encore dans des zones de recrû d'épineux (dongaare, fflde). Il s'agit d'une chasse de proximité, voire d'une chasse domestique, une partie des termitières étant perçues comme appropriées. Cette chasse repose sur une forme de cooptation en petits groupes. Toutefois ces mêmes chasseurs de termitières, rappelons-le, participent également aux chasses collectives de leurs villages. Chez les Giziga la chasse est organisée à partir de la chefferie bwi Marva ou des chefferies qui en sont issues. Il existe encore aujourd'hui un chef de chasse (bwi madala) pour l'ensemble du pays giziga, originaire au départ du clan ngoyang ${ }^{14}$. Il doit être obligatoirement non musulman. Les chasses collectives à la lance et au bâton de jet ont précisément lieu le vendredi. Luimême en désigne chaque fois la direction. Son rôle est de veiller au respect des codes de chasse et qu'au retour les rites de purification de la brousse soient strictement accomplis. 
Il revêt un rôle d'arbitre dans les différentes chasses collectives de village. Après les récoltes il dirige une fête importante au mois de la chasse (kiya nguu madala), la fête c udavo. Il "ranime» pour la nouvelle campagne les flûtes de chasse qui avaient été remisées après la précédente et qu'il avait fait placer pour les enfumer avec des feuilles de burkalaf (Combretum glutinosum) ${ }^{15}$. Les rituels concernant la chasse restent encore bien vivants chez les Giziga bwi Marva.

Depuis longtemps les Giziga sont en contact avec des chasseurs bornouans (kirmina) qui se revendiquent gaw, l'aristocratie de la chasse. Au nord de Maroua existent encore des villages de ces gaw qui allaient chasser dans les zones tampons vides, très giboyeuses entre le royaume du Wandala et les principautés peules: Adiya, Walassa, Ngaba, Massourdouba, Méhé et Joundé plus dans la plaine (Seignobos 2011: 36). Ces communautés de chasseurs ont prospéré au $\mathrm{XIX}^{\mathrm{e}}$ siècle et plus encore pendant la période coloniale. Les Giziga ont pu servir d'adjoint (suuka) à ces gaw, partageant leur campagne de chasse dans les yaérés ${ }^{16}$ et convoyant les lanières de viande séchée transportées à dos d'âne. Ils devaient recevoir les enseignements de cette mystique liée à la brousse avec tout le compendium des comportements à tenir pour que la chasse soit productive et maints « remèdes » de protection dits matapurum. Car l'enseignement des gaw s'attache à dominer la brousse et à s'en prémunir. L'apprentissage des traces, les techniques d'approche du gibier, le comportement des animaux selon les saisons, les aliments préférentiels... leurs sont acquis. Pour les chasseurs de termitière en revanche c'est là l'essentiel des connaissances qu'ils transmettent à leurs fils ou à leurs " porteurs de sac». Les termitières elles-mêmes, en particulier les plus hautes (waande hippere, fflde) ne sont pour les gaw qu'un lieu sacrificiel pour se débarrasser d'envoûtements après de complexes ablutions.

71 Revenus dans leurs propres espaces de chasse villageois les Giziga ne font plus grand cas de cet enseignement et reprennent leur propre bestiaire animalier, qui n'est pas non plus dépourvu de shetene. Ainsi la venue de serpents ayant provoqué des décès les fait s'interroger sur leur nature. Puisqu'ils chassent les rats du Nil, ne serait-ce pas ces derniers qui «mystiquement » auraient convoqué le cobra tueur? Ne s'agit-il pas d'un «tandem mystique» qu'il y a lieu d'exorciser? Le masahay intervient pour un « retournement » de cette attaque occulte vers ceux censés en avoir été l'origine. Avec la conscience d'appartenir à deux mondes différents, les notables giziga, et le chef de chasse en particulier, se montrent très opposés aux gaw. Les Giziga cherchent à garder la maitrise de leur brousse, ils dénoncent les pratiques des chasseurs bornouans qui mènent des chasses individuelles ou par équipes sous la conduite de leur kacalla ${ }^{17}$ indépendamment des ritualistes giziga. Ils s'accusent mutuellement de concurrence et d'entrainer un dérèglement de leurs pratiques de chasse respectives. Le chef bwi Marva, reprenant les accusations des gaw d'avoir « fermé la brousse " (mabbugo ladde en fflde) convoque les gaw leur intimant de cesser leurs manigances coupables tout en interdisant la présence de gaw étrangers sur son territoire.

Depuis les années 1990 les gaw subissent un véritable déclassement social par l'inexorable recul de la grande faune qui les pousse à être surtout des tradipraticiens et face à un islam citadin de plus en plus prompt à dénoncer leurs pratiques ésotériques. Aussi sont-ils devenus moins scrupuleux dans leur art de la chasse, reprenant certaines techniques auparavant décriées, de même pour leur recrutement. Ils n'en poursuivent pas moins une sorte de mise sous tutelle de ces chasseurs subalternes, sans toutefois les contraindre à s'islamiser. Ils leur prodiguent encore leurs savoirs, des formes de «blindages", 
«d'anticorps » et autres recettes de protection... contre une modique taxe en peaux de varans et de serpents qu'ils commercialisent auprès des artisans maroquiniers de Maroua.

Les derniers maîtres gaw comme Bappa de Ginadji, héritier de Gaw Mallum de Massourdouba, tous kacalla fameux, livrent encore leur enseignement sous la forme " classique », les griots propres aux guildes de chasseurs ayant quasi disparu. Il repose sur des biographies voire des hagiographies comme celle que l'on pourrait appeler «les riches heures de Kacalla Goumbo Abaca » ou encore des biographies critiques, « la faute du gaw Hassana ", dans ces récits moraux, dont certains semblent fort anciens, sont exposés les bons et les mauvais comportements du chasseur.

en récits d'événements n'en continuent pas moins de nos jours. En 1998, Kacalla Bappa a pris comme suuka, Sarman Gaso, un Masa païen à qui il enseigne son art. Dans une termitière, sous un tamarinier, il y avait un énorme cobra désigné par Bappa comme le « fils de l'arbre ». Sa capture devait se faire avec un maximum de précautions rituelles et il ne fallait pas être isolé dans cette entreprise. Sarman décida d'intervenir seul. Il enfuma la termitière mais l'arbre faillit brûler et lui avec sans que le serpent ne sorte. Sarman fut ensuite attaqué par le shetene de la termitière. Il devint fou et alla se prendre à un autre tamarinier. Moralité : il convient, pour le suuka de toujours rester sous la protection de son maitre «baaba lagawal ", le père de l'arc, jusqu'à son émancipation. Chez les gaw le maître maintient longtemps son emprise sur ses burza, disciples/élèves, avant qu'ils ne bénéficient, à leur tour, du statut de gaw. Cet enseignement est-il encore audible aujourd'hui?

Les chasseurs de termitières giziga ont acquis auprès des chasseurs professionnels bornouans un savoir "théorique " de la chasse qu'ils sont capables d'énoncer à tout moment, mais ils ne le suivent que très occasionnellement. Ils déclarent, par exemple, qu'avant toute campagne de chasse il faut sacrifier un hérisson placé au centre d'un cercle de chasseurs et de tirer augure de la position de ses pattes. Le hérisson serait aux chasseurs ce qu'est la gallinomancie aux villageois. Mais les chasseurs giziga ne le pratiquent pas. Lorsque le gibier a été tué, il faut se livrer à différents rituels tirés de leur pratique, mais c'est exceptionnellement qu'ils en réalisent un succédané et sur un gibier plus gros que les autres. Les chasseurs giziga bwi Marva ne sont pas prêts à s'inféoder à ces guildes finissantes de chasseurs bornouans, mises à mal par l'islam intégriste qui s'empare de la région. Sur ces piémonts des Mandara où, comme se lamentent les chasseurs, la «biche », nom en français local de la « gazelle» (Gazella rufifrons), a quasi disparu, ils préfèreront toujours la gestion routinière de leurs termitières qui n'en représentent pas moins des sortes de refuges pour les derniers représentants de la faune sauvage.

\section{BIBLIOGRAPHIE}

Dobigny G., Tatard C., Kane M., Gauthier Ph., Brouat C., Ba K. \& Duplantier J.-M. 2011 - A cytotaxonomie and DNA-based survey of rodents from Northern Cameroon and Western Chad. Mammalian Biology 76 (4) : 417-427. 
Dounias E. 2016 - Des moissons éphémères. L'art de collecter et de consommer les termites sous les tropiques. In : Motte-Florac E. \& Le Gall P. (Ed.) Savoureux insectes, de l'aliment traditionnel à l'innovation gastronomique. Presse université de Rennes/IRD : 273-339.

Granjon L. \& Duplantier J.-M. 2009 - Les rongeurs de l'Afrique sahélo-soudanienne. Marseille, IRD Éditions/Publications scientifiques du Muséum, 215 p.

Jaouen R. et al. 1998 - Dictionnaire giziga sud-français. Polygraphie, Maroua, 190 p.

Seignobos C. 2011 - Les gaw du Cameroun, de chasseurs à tradipraticiens. Journal des Africanistes $81(1): 35-59$.

Seignobos C. 2014 - La chasse/pêche aux batraciens : aux origines de la vie des populations du bassin du lac Tchad ? (l'exemple du Diamaré, Cameroun). Anthropozoologica 49 (2) : 305-325.

Seignobos C. 2015 - Des insectes et des hommes (Nord du Cameroun). Revue d'Ethnoécologie 7/2015 Varia. https://journals.openedition.org/ethnoecologie/2180.

Seignobos C., Deguine J.P. \& Aberlenc H.P. 1996 - Les Mofu et leurs insectes. Journ. d'Agric. Trad. et de Bota. Appl. 38 (2) : 125-187.

Seignobos C. \& Tourneux H. 2002 - Le Nord-Cameroun à travers ses mots, dictionnaire de termes anciens et modernes. Paris, IRD Éditions, Karthala, 334 p.

\section{NOTES}

1. Mayo signifie rivière en foulfouldé et accompagne leur nom dans tout le Nord du Cameroun

2. Nos principaux informateurs, les villageois avec qui nous avons réalisé plus de trois entretiens, Bappa, giziga medemtere du village de Dogba: Sadjo, giziga de Goumtokoy; Sali Bouba de Mokora, Hamidou Angokay de Djébé ; Mana Kari, Ndjidda Kitikil, Assoumbay Katchala, tous trois de Kosséwa. Parmi les chasseurs spécialisés : Kani Abiiko, chasseur de varans et de serpents, Abba Akouli, chasseur de petits carnassiers. Maliki Wassili de Maroua/Kosséwa fut notre principal interprète et enquêteur.

3. Les enquêtes se sont déroulées entre 1995 et 2010, pour la plupart chez des Giziga bwi Marva et les appellations retenues sont données dans cette langue. Toutefois la langue véhiculaire de la région étant celle des Peuls, nous citerons également des appellations avec la mention fflde (foulfouldé)

4. Dictionnaire sous forme ronéo.

5. Certains informateurs hésitent aussi avec une autre pratique liée à des campagnes de chasse aux anoures dans les mêmes endroits. On avait alors besoin d'eau à chauffer pour les tuer ( Pyxicephalus edulis, Ptychadena spp.) à travers des vanneries ou des sacs, sans détériorer leurs chairs, avant de les mettre à sécher au soleil ou de les fumer (Seignobos 2014).

6. Sur les massifs-îles en avant des monts Mandara, à Mékéri, Griya, Molkwo, les femmes exploitent les termitières (Bellicositermes sp.) en particulier pour leurs poussins. Elles attirent les termites en bourrant de bottes de tiges de niébés (Vigna unguiculata) des poteries retournées, légèrement enfoncées par le col à la périphérie de ces termitières. Le dispositif dépasse rarement quatre à cinq canaris (grandes poteries) disposés à égale distance les uns des autres. Les pièges se relèvent en les retournant, les termites sont alors ramassés au fond, à la louche. Ainsi les femmes nourrissent-elles la termitière tout en prélevant un lot constant de termites. Ces prélèvements s'effectuent tout au long de la saison sèche et durant des années.

7. Nous remercions Gauthier Daubigny (IRD) pour les identifications et notes qu'il nous a communiquées après ses missions dans le nord du Cameroun. Son rabatteur et principal interprète dans la région de Maroua était Maliki Wassili. (Dobigny et al. 2011) 
8. Munndul est un terme d'origine kanuri qui désigne le trou du bac du tanneur que l'on referme après usage.

9. Est-ce le circaète brun ou siilde (Milvus migrans), le milan noir, qui peut le prendre la nuit dans ses serres et le laisser choir du ciel avant de le dévorer?

10. Un minéral composé de carbonate et de sodium hydraté.

11. Les groupes non musulmans de la plaine, Mundang, Gidar, Tupuri, Masa, possèdent des bâtons de jet quasi identiques.

12. Chez des montagnards comme les Mafa, le python est protégé pour lutter contre les rongeurs. Un grand pourcentage d'enclos (gay) l'élèvent même et le laissent prospérer sous le rebord des toitures. Chez les Mofu voisins des Giziga, la graisse de python est recherchée pour oindre les pierres de pluies. Aussi défend-on de tuer le python lors de périodes de sécheresse. De nombreuses chansonnettes ou des mélopées sont entonnées pour l'encourager à sortir de son terrier et lorsqu'on le tue, on s'excuse de le faire et on le flatte en lui chantant shishe mabar (honneur à toi lion).

13. Pour rédiger cette partie, je me suis adressé à d'autres cercles de chasseurs giziga et bornouans. Abaaji Goni, Bornouan de Ginadji, Ali Abatcha, Bornouan du village de Massourdouba, Bouba Amadou, Foulbéïsé, de Yidi Wadi, près de Pété et Bello Kedde, giziga islamisé. Hamadou Soba et Yougouda de Tambajam, islamisés d'origine servile ; Bouhari Djoulé, giziga animiste, et Adama Garga, Giziga chrétien de Kosséwa.

14. Notre informateur Oumarou Asoumday, de Djébé, du clan Gorey apparenté au clan Ngoyang, était chef de chasse du pays giziga en 1994, lors de mes enquêtes concernant les structures de la chefferie bwi Marva et son fonctionnement.

15. Ces feuilles servent à soigner des plaies et à purifier les silos par la fumée et à en chasser les déprédateurs.

16. Du ffde yaayre, désigne au Cameroun les plaines d'inondation (Seignobos \& Tourneux 2002 : 280).

17. Le kacalla fait partie de la titulature bornouane. Il désigne ici le chef de guilde de chasse.

\section{RÉSUMÉS}

Creuser et enfumer les terriers n'a jamais été une chasse valorisée parmi les communautés du nord du Cameroun, musulmanes ou non. Elle reste celle d'agriculteurs appartenant aux classes d'âge adultes exploitant les entours des parcellaires de leurs terroirs.

Parmi eux, les chasseurs de termitières, ici les Giziga de la région de Maroua, n'en forment pas moins une catégorie à part qui associe un savoir sur les termitières vives exploitées une fois l'an, lors de l'essaimage des adultes sexués ailés, et celles des termitières mortes, abandonnées par leur colonie de termites et devenues le refuge d'une faune diverse, à poils, à piquants ou à écailles. Il convient alors, pour les chasseurs, de connaître finement l'éthologie et la « sociologie » de ces colocataires de termitières.

Le constant recul du gibier, non seulement sur les piémonts des monts Mandara, mais aussi dans la plaine du Diamaré attenante fait de cet univers de chasse, autrefois marginal, l'un des rares encore productifs.

Digging and smoking animals out of their holes have never been considered valuable hunting techniques in Northern Cameroonian communities, either Muslim or other. They are used by 
adult farmers using the surrounding areas of their plots.

Among them, termite mounds' hunters - namely here, the Gizigas from the Maroua region remain a separate category associating knowledge about living termite mounds exploited once a year, during sexually matured winged adults' swarming, and knowledge about dead termite mounds that have been given up by their colonies and become the refuge of a diverse, furry, priclkly and scaly animals. The hunters thus need to know many details about the ethology and "sociology" of the termite mounds' roommates.

The constant decrease of game, on the piedmonts of Mounts Mandara as well as in the adjacent Damaré plains, is making this formerly marginal hunting territory one of the rare ones that is still attractive today.

INDEX

Keywords : termite mound, small game, hunting, North Cameroon, Maroua region, Bornouans Population Giziga Index géographique : Cameroun

Mots-clés : termitière, petit gibier, chasse, nord du Cameroun, région de Maroua, Bornouans

\section{AUTEUR}

\section{CHRISTIAN SEIGNOBOS}

Géographe, directeur de recherche émérite IRD 